\title{
A Study of Synergistic Antibacterial Activity of Silver Nanoparticles with Antibiotics against Multi-Drug Resistant Pseudomonas aeruginosa in Intensive Care Units of a Tertiary Care Hospital
}

\author{
Komal Mirlekar*, Anjali Swami, Sujata Baveja and Shanta Shubhra Das \\ Department of Microbiology, Lokmanya Tilak Municipal Medical College \& GH, Sion, \\ Mumbai, Maharashtra - 400 022, India \\ *Corresponding author
}

\begin{abstract}
A B S T R A C T
\section{Ke y words}

Synergistic activity

Nanoparticles with

antibiotics, Multi-drug

resistant organisms

Article Info

Accepted:

04 October 2018

Available Online:

10 November 2018

Evolution of multi drug resistant isolates has become rampant in today's world. The credit of this goes to the incomplete dosage and irrational use of antibiotics. The human brains are now in search of alternate methods. Use of Silver nanoparticles is one amongst them. In the following study, 50 multi-drug resistant clinical isolates of $P$. aeruginosa acquired from the intensive care units were tested with antibiotics, AgNps and AgNp-antibiotic conjugates. The conjugates were prepared by impregnating $30 \mu \mathrm{l}$ of AgNps in sterile plain disks and the antibiotic disks separately. The antibiotics tested were Ciprofloxacin, Amikacin, Ceftazidime and Piperacillin-Tazobactam. Maximum number of isolates showed zone enhancement with both $\mathrm{AgNp}$ and $\mathrm{AgNp}$ - antibiotic conjugates. Fewer numbers of isolates that did not show enhancement may have different mechanisms of resistance like point mutations. Nanoparticles are not able to overcome point mutations. Thus, Nanotechnology has served to be a boon to overcome the massive war against Multidrug resistant isolates.
\end{abstract}

\section{Introduction}

Bacterial infections are the major cause of mortality and morbidity in today's world. Wide spread and haphazard use of antibiotics increase the emergence of multi-drug resistant bacteria. The treatment of these infections has become a public health concern due to bacterial resistance to antibiotics. $P$. aeruginosa and $A$. baumannii are the major nosocomial pathogens, responsible for healthcare-associated infections and hospital outbreaks, with multidrug resistance to antimicrobial agents i.e. cephalosporins, fluoroquinolones, aminoglycosides, polymyxins and even carbapenems. A possible strategy to control the resistant microorganisms is to improve the biocide substances activity against them. Many scientific journals are giving warning signals about antibiotic resistance.

The man in his quest for knowledge has been conceiving and developing physical world and its components in bigger than the biggest and smaller than the smallest dimensions of mass, length and time. The technology that deals with the materials ranging from 1 to $100 \mathrm{~nm}$ is 
termed as Nanotechnology. Gold nanoparticles, Silica nanoparticles, Silver nanoparticles and Quantum dots have found wider applications in diagnosis and therapeutics (Nafeesa Khatoon et al., 2017; Ge et al., 2014; Naqvi et al., 2013). Silver nanoparticles have become popular in the industry and medicine due to its antibacterial, antifungal, larvicidal and anti-parasitic characters. They have been extensively used for diagnosis, drug delivery, treatment, wound dressings and contraceptive devices. On conversion of Silver into its nano form, increases its surface area to volume ratio, thus the physical, chemical and biological properties of Silver are enhanced. These distinct physical properties of $\mathrm{AgNps}$ like conductivity, chemical stability, catalytic activity and nonlinear optical behavior are a matter of interest. The difference in size, shape and morphology had a tremendous impact on the applications of the nanoparticles. The evolution of multi drug resistant organisms has incited alternate strategies to control infections. Several enzymes and mutations in genomic arrangements accelerate multidrug resistance by modifying the efflux pump from the cells, which thereby decreases the activity of bacteria to antibacterial agents. The last decade, witnessed the emergence of various Nano conjugates against pathogenic microbes (Naqvi et al., 2013). The nanoparticleantibiotic conjugates reduce the quantity of both agents in the dosage, which reduces their toxicity and increases antimicrobial properties. Several recent studies indicated that Silver nanoparticles may strengthen the antibacterial effects of antibiotics, either additively or synergistically. Unfortunately, the synergistic effects of Silver nanoparticles-antibiotics combinations were proven mostly for susceptible bacteria (Panáček et al., 2016). Only a few studies confirmed a successful synergistic effect of Silver nanoparticles antibiotics combinations in resistant bacteria.
In the present study, silver nanoparticles were tested against multi-drug resistant Gram negative clinical isolates of Pseudomonas aeruginosa as there are very few studies on the synergistic effect of Silver nanoparticles on these multi-drug resistant organisms. The study was done from the period of January 2017 to May 2017.

\section{Materials and Methods}

\section{Preparation of inoculum}

In the present study, 50 multidrug resistant isolates of Pseudomonas aeroginosa from clinical samples were tested. These pathogens were isolated from blood (25), endotracheal secretions (15) and pleural fluid (10) in ICU patients of a tertiary care hospital. All these isolates were resistant to four classes of drugs, i.e. fluoroquinolones, aminoglycosides, $\beta$ lactam- $\beta$ lactamase inhibitor and cephalosporins. The specific antibiotics used were Ceftazidime, Amikacin, Ciprofloxacin and Piperacillin - tazobactam.

\section{Preparation of silver nanoparticles}

Polyvinylpyrrolidone (PVP) capped AgNp's were procured from Institute of Chemical technology, Mumbai, India. An AgNP suspension of $100 \mathrm{ppm}$ was prepared by dissolving $0.1 \mathrm{mg}$ of chemically synthesized AgNps in $1 \mathrm{~mL}$ autoclaved deionized water. The suspension was sonicated for 20 minutes to avoid deposition of AgNps. $30 \mu \mathrm{l}$ of $\mathrm{AgNp}$ suspension was used in the study.

\section{Preparation of discs}

AgNp suspension was calibrated by automated pipette with sterile tips. The tips were sterilized in the autoclave at $121^{\circ} \mathrm{C}$ for 15 mins. $6 \mathrm{~mm}$ sterile discs were obtained from Himediapvt ltd. Soon after sonication, $30 \mu \mathrm{l}$ of this suspension was inoculated immediately in 
the sterile discs. Antibiotic disks of Ciprofloxacin, Amikacin, Piptaz, and Ceftazidime were obtained from Himediapvt ltd. These antibiotic discs were inoculated with $30 \mu \mathrm{l} \mathrm{AgNp}$ suspension to check the synergistic effect of $\mathrm{AgNp}$ with the antibiotics.

\section{Procedure}

The suspension of all these isolates was prepared by diluting with $0.9 \% \mathrm{NaCl}$ solution. The inoculum was plated on Muller Hinton agar plates after matching with 0.5 McFarlands reagent. 5 disks were placed equidistantly from each other, $15 \mathrm{~mm}$ away from the edge of the plate. These 5 disks include the 2 disks of antibiotics, 1 nanoparticle disk and 2 disks of AgNpantibiotic conjugate. The plates were kept overnight in the incubator at $37^{\circ} \mathrm{C}$. The zone of inhibition was measured the next day.

\section{Statistical Analysis}

Statistical analysis was performed using IBM Statistical Package for the Social Sciences version 20 (SPSS v20, IBM). Categorical variables between two groups were compared using Chi-square test and Fisher's exact test. A $p$ value of $<0.05$ was considered significant.

\section{Results and Discussion}

Multi drug resistance (MDR) can be defined as resistance to at least four classes of antibiotics. Emergence of MDR strains is often due to selective pressure of antimicrobial therapy. These drug resistant pathogens are more pathogenic with high mortality rate than that of wild strain. The scope of interest of the present study was exploring the capabilities of Silver nanoparticles alone and also in conjugation with various antibiotics against multidrug resistant clinical isolates. The efficacy of antibiotics, Silver nanoparticles, and their combination in terms of zone of inhibition was measured against 20 different isolates of Pseudomonas aeruginosa. Ceftazidime $+\mathrm{AgNp}$ for Pseudomonas aeruginosa reported the highest antibacterial activity. The least synergistic antibiotic activity was reported for Amikacin $+\mathrm{AgNp}$.

The results of the synergistic effect in the form of fold increase (Qasim et al., 2014) are presented in Table 1.

Pseudomonas aeruginosa is an opportunistic pathogen capable of causing nosocomial infections. It is an important multi-drug resistant pathogen in Intensive care set up leading to higher morbidity and mortality. Also $P$. aeruginosa is a scourge of hospital burn units. This is also justified by various studies (Singh et al., 2014). In the present study, Pseudomonas aeruginosa was tested for its three Group A and one Group B drug (CLSI 2017) Ciprofloxacin, Piptaz, Ceftazidime and Amikacin respectively.

Ciprofloxacin is a 2nd generation fluoroquinolone. It is Group A drug for Pseudomonas aeruginosa (CLSI, 2017). Ciprofloxacin-AgNp combination gives better results than Ciprofloxacin alone. More than half 28/50 (56\%) of the Pseudomonas isolates reported zone enhancement with a considerable fold area increase of $45.27 \%$. Similar kind of observations was reported by Naqvi SZH in his study (Naqvi SZH et al., 2013). Amikacin is an aminoglycoside, Group B drug for Pseudomonas aeruginosa (CLSI 2017). In case of Amikacin, fewer isolates i.e. 22/50 (44\%) exhibited synergism. The fold area increase was also on the lower side, i.e. $41.91 \%$. Similar kind of observations was reported by Barapatre A et al., (Barapatre A et al., 2016). Barapatre also proposed that the Ag-NPs might be disrupting the bacterial cell wall structure and surface charge balance, which eventually change the permeability of 
cell wall of Pseudomomas aeruginosa due to which antibiotics have better opportunity to approach the individual bacterial cell. According to the report of the NIHCDC, about 65-80 \% infections occurred by biofilm formation microbes, amid which the Gramnegative bacterium $P$. aeruginosa, E. coli, and the Gram-positive Staphylococci, S. aureus are the most common ones. The antibacterial activity of Piptaz for Pseudomonas aeruginosa is reported in Table 1 (CLSI, 2017).

Piptaz is a Group A drug for Pseudomonas aeruginosa. 32/50 (64\%) of isolates reported zone enhancement with fold area increase $75.88 \%$. Similar observations were reported by Ekbal H. Abdel Hafez et al., (2017). The antibacterial activity of Ceftazidime for Pseudomonas aeruginosa is reported in Table 1. Ceftazidime is a third-generation cephalosporin, Group A drug for Pseudomonas aeruginosa (CLSI 2017). 30/50 (60\%) of isolates of Pseudomonas aeruginosa those were resistant to Ceftazidime showed zone enhancement in presence of AgNps with increase in fold area of $87.77 \%$. Similar observations were reported by Richa Singh $e t$ al., (Richa Singh et al., 2017). Thus, the study was statically significant for all the tested antibiotics in Pseudomonas aeruginosa. A recent study by Morones-Ramirez et al., described the effects of silver ions and their synergy with antibiotics to treat a range of infections both in vivo and in vitro (MoronesRamirez et al., 2013). Their results demonstrated that silver ion-antibiotic combinations increased the production of reactive oxygen species, which in turn increased the membrane permeability of Gram-negative bacteria. The authors hypothesized this may potentiate the activity of a broad range of antibiotics against Gramnegative bacteria in different metabolic states, as well as restoring antibiotic susceptibility to resistant bacterial strains. This synergistic effect and the results for the capped AgNps were also reported by Markowska et al., (2014) (Table 2).

Table.1 Comparison between mean zone sizes of AgNps, antibiotic and conjugates

\begin{tabular}{|l|l|}
\hline AgNp & $\mathbf{1 0 . 8} \pm \mathbf{8 . 4 8}$ \\
\hline Ciprofloxacin & $7.4 \pm 4.16$ \\
\hline Ciprofloxacin + NP & $10.75 \pm 4.18$ \\
\hline Fold increase & $45.27 \%$ \\
\hline P value & $<0.001$ (very very Significant) \\
\hline Amikacin & $6.8 \pm 3.34$ \\
\hline Amikacin + NP & $9.65 \pm 9.5$ \\
\hline Fold increase & $41.91 \%$ \\
\hline P value & $<0.001$ (very very Significant) \\
\hline Ceftaridime & $6.95 \pm 4.18$ \\
\hline Ceftaridime+ NP & $13.05 \pm 12.74$ \\
\hline Fold increase & $\mathbf{8 7 . 7 7 \%}$ \\
\hline P value & $<0.001$ (very very Significant) \\
\hline Piptaz & $7.05 \pm 3.52$ \\
\hline Piptaz + NP & $12.4 \pm 10.82$ \\
\hline Fold increase & $\mathbf{7 5 . 8 8 \%}$ \\
\hline P value & $<0.001$ (very very Significant) \\
\hline
\end{tabular}


Table.2 Number of isolates with zone enhancement

\begin{tabular}{|l|l|}
\hline Ciprofloxacin + NP & 28/50 (56\%) \\
\hline Amikacin + NP & $22 / 50(44 \%)$ \\
\hline Piptaz + NP & $32 / 50(64 \%)$ \\
\hline Ceftaridime+ NP & $30 / 50(60 \%)$ \\
\hline
\end{tabular}

These data demonstrate the ability of small PVP-capped AgNps to synergistically enhance the antimicrobial effects of antibiotics against $P$. aeruginosa biofilms in vitro, and they support a potential role for these small nanoparticles in reducing the antibiotic burden of patients treated for chronic infections.

A synergistic effect of antibiotics in conjugation with biologically synthesized AgNps increased the susceptibility among the tested bacteria from $20 \%$ to $30 \%$. The combined effect of $\mathrm{AgNps}$ and antibiotics was notable against these isolates of $P$. aeruginosa.

Nanotechnology provides a good platform to overcome the problem of resistance, with the help of the silver nanoparticles. Altogether, combining antibiotics with $\mathrm{AgNps}$ provides one potential approach to an effective fight against the unresolved problem of an increasing resistance of pathogenic bacteria against traditional antibiotics. The three fundamental mechanisms of antimicrobial resistance are (1) enzymatic degradation of antibacterial drugs, (2) alteration of bacterial proteins and (3) changes in membrane permeability to antibiotics. The silver nanoparticles not only alter the expression proteinases but also suppress tumour necrosis factor (TNF), IL-12 and IL- $1 \mathrm{~b}$ and induce apoptosis of inflammatory cells Moreover, silver nanoparticles are also responsible for cytokine modulation in wound healing and inhibition of the biofilm formation (Rai et al., 2012). The probable mechanism involved in enhanced antibacterial activity of antibiotics with silver nanoparticles may be attributed to the bonding reaction between nanoparticles and antibiotics. Then the antibiotic-silver nanoparticle combination may attach on the cell membrane result in cell wall lysis, which was followed by the entry of AgNps-antibiotic combination into the cell and may result in the DNA unwinding leads to cell death. It was also found that the silver nanoparticles enhanced the reaction rates of antibiotics in a synergistic mode as well as in its own way on different kinds of pathogens.

In the present study, few isolates reported low significance in comparison to the other isolates. One of the mechanisms of resistance to cephalosporins and fluoroquinolones in Pseudomonas is point mutations in the bacterial targets gyrA and parC topoisomerase enzymes. It is possible that AgNps are not able to act on the genetic level to overcome the point mutations. Ceftazidime-AgNp combination reported the highest zone of inhibition of Pseudomonas aeruginosa. Thus, antibacterial activity of $\mathrm{AgNp}$ and AgNp-antibiotic conjugates are helpful in those MDR isolates in which the resistance mechanism is other than point mutations. Instead of costlier version of $\mathrm{AgNps}$ that are commercially available, they can be prepared in the laboratory by the any of the suggested methods. These methods are cost effective. The following study used $30 \mu \mathrm{l}$ of AgNps, studies should be done using different concentrations of AgNps and antibiotics. It is likely that higher concentrations of AgNps might work for Acinetobacter species. The potential toxicity of $\mathrm{AgNp}$ is lower than the antibiotics. The synergistic action of antimicrobial agents can reduce the need for high dosages and minimize side effects.

\section{References}

Barapatre A, Aadil KR, Jha H. Synergistic antibacterial and antibiofilm activity of silver nanoparticles biosynthesized by 
lignin-degrading fungus. Bioresource Bioprocess [Internet]. 2016; 3(1):8. Available from: http://www. bioresourcesbioprocessing.com/content/3/ $1 / 8$.

Ge L, Li Q, Wang M, Ouyang J, Li X, Xing MMQ. Nanosilver particles in medical applications: Synthesis, performance, and toxicity. Int J Nanomedicine. 2014; 9(1): 2399-407.

Hafez EHA, Ahmed EA, Abbas HS, Salah RA, Din E. Efficacy of Antibiotics Combined with Biosynthesized Silver Nanoparticles on some Pathogenic Bacteria. Int J Sci Res. 2017; 6(1):1294-303.

Markowska K, Grudniak AM, Krawczyk K, Wróbel I, Wolska KI. Modulation of antibiotic resistance and induction of a stress response in Pseudomonas aeruginosa by silver nanoparticles. J Med Microbiol. 2014; 63(PART 6):849-54.

Morones-Ramirez JR, Winkler JA, Spina CS, Collins JJ. Silver Enhances Antibiotic Activity Against Gram-Negative Bacteria. Sci Transl Med [Internet]. 2013; 5(190):190ra81-190ra81. Available from: http://stm.sciencemag. org/cgi/doi/10.1126/scitranslmed.300627 6.

Nafeesa Khatoon, Jahirul Ahmed Mazumder and Meryam Sardar. Biotechnological Applications of Green Synthesized Silver Nanoparticles. J Nanosci Curr Res., 2017, 2:1.

Naqvi SZH, Kiran U, Ali MI, Jamal A, Hameed A, Ahmed S, et al., Combined efficacy of biologically synthesized silver nanoparticles and different antibiotics against multidrug-resistant bacteria. Int $\mathbf{J}$ Nanomedicine. 2013; 8: 3187-95.

Panáček A, Smékalová $M$, Večeřová $R$, Bogdanová $\mathrm{K}$, Röderová $\mathrm{M}$, Kolář $\mathrm{M}$, et al., Silver nanoparticles strongly enhance and restore bactericidal activity of inactive antibiotics against multiresistant Enterobacteriaceae. Colloids Surfaces B Biointerfaces [Internet]. 2016; 142: 3929. Available from: http://dx.doi.org/10.1016/j.colsurfb.2016. 03.007.

Qasim M, Lim D-J, Park H, Na D. Nanotechnology for Diagnosis and Treatment of Infectious Diseases. $\mathbf{J}$ Nanosci. Nanotechnol [Internet]. 2014; 14(10):7374-87. Available from: http://openurl.ingenta.com/content/xref?g enre $=$ article $\&$ issn $=1533-4880 \&$ volume $=14 \&$ issue $=10 \&$ spage $=7374$.

Rai MK, Deshmukh SD, Ingle AP, Gade AK. Silver nanoparticles: The powerful nanoweapon against multidrug-resistant bacteria. J Appl Microbiol. 2012; 112(5):841-52.

Singh K. Antibacterial Activity of Synthesized Silver Nanoparticles from Tinosporacordifolia against Multi Drug Resistant Strains of Pseudomonas aeruginosa Isolated from Burn Patients. J Nanomed Nanotechnol [Internet]. 2014; 5(2). Available from: https://www.omicsonline.org/openaccess/antibacterial-activity-synthesized silver-nanoparticles-tinosporacordifolia-multi-drug-resistant-strainspseudomonas-aeruginosa-isolated-burnpatients-2157-7439-5-192.php?aid=25 859.1

Singh R, Wagh P, Wadhwani S, Gaidhani S, Kumbhar A, Bellare J, et al., Synthesis, optimization, and characterization of silver nanoparticles from Acinetobacter calcoaceticus and their enhanced antibacterial activity when combined with antibiotics. Int J Nanomedicine. 2013; 8: 4277-90.

\section{How to cite this article:}

Komal Mirlekar, Anjali Swami, Sujata Baveja and Shanta Shubhra Das. 2018. A Study of Synergistic Antibacterial Activity of Silver Nanoparticles with Antibiotics against Multi-Drug Resistant Pseudomonas aeruginosa in Intensive Care Units of A Tertiary Care Hospital. Int.J.Curr.Microbiol.App.Sci. 7(11): 83-88. doi: https://doi.org/10.20546/ijcmas.2018.711.012 